\title{
El Jardín Etnobiológico de
Hidalgo Maximino
Martínez
} Consuelo Quevas-Cardona y María Teresa Pulido Silva Universidad Autónoma del Estado de Hidalgo cuevas@uach.edu.mx mtpulido@uaeh.edu.mx orcid.org/0000-0001-8988-097X orcid.org/0000-0003-1307-9574

Recibido: 15 de octubre de 2020 Aceptado: 7 de noviembre de 2020

\section{Resumen}

El Consejo Nacional de Ciencia y Tecnología ha echado a andar un proyecto que busca establecer jardines etnobiológicos en cada estado del país, lugares en los que se buscará unir los conocimientos científicos con los tradicionales, debido a que actualmente se sabe que los pueblos originarios del mundo son los que han logrado conservar la biodiversidad, hecho reconocido por biólogos y epistemólogos ambientales de todo el mundo. Nuestra Universidad obtuvo el apoyo para establecer el correspondiente al estado de Hidalgo, que tendrá dos sedes, una en Tlanchinol y otra en Pachuca, la primera en medio de la exuberancia del bosque mesófilo de montaña y la segunda en una zona de matorral xerófilo.

Palabras clave: Conservación simbiótica, intercambio de saberes, conservación in situ y ex situ.

\section{Abstract}

The Mexican National Council of Science and Technology has launched a project with the goal of establishing ethnobiological gardens in each of Mexico's states. These gardens will seek to unite scientific knowledge with traditional knowledge, motivated by the fact, now recognized by biologists and environmental epistemologists worldwide, that it is the native people of the world who have managed to conserve biodiversity. Our university has obtained support to establish the ethnobiological garden for the state of Hidalgo. It will be located in two sites, one in Tlanchinol in the lush montane cloud forest and the other in Pachuca, in a xerophilic scrub zone.

Keywords: Symbiotic conservation, knowledge exchange, in situ and ex situ conservation.

A fines de 2019 Conacyt dio a conocer la propuesta de crear 32 jardines etnobiológicos en el país, uno en cada estado. La Universidad Autónoma del Estado de Hidalgo sometió la propuesta de establecer el del estado de Hidalgo que tendrá dos sedes, una situada en Tlanchinol y la otra en el Parque Científico y Tecnológico de nuestra Universidad, en Pachuca. El Jardín Etnobiológico de Hidalgo Maximino Martínez recibirá este nombre en honor de un botánico hidalguense, reconocido nacional e internacionalmente por sus aportes a la etnobiología. Fue profesor normalista y botánico autodidacta, precursor de la botánica económica y la exploración de los recursos vegetales de México. Entre sus mayores logros está el haber sido fundador de la Sociedad Botánica de México (presidente de 1941 a 1944, secretario de 1944 hasta su muerte en 1964), así como la publicación de más de 100 obras, incluyendo su Catálogo de nombres vulgares y científicos de plantas mexicanas, la cual es la única fuente en idioma español citado por Nikolái Vavilov en su estudio clásico sobre los centros de origen de las plantas domesticadas en el mundo.

Los jardines etnobiológicos tienen como fin ser espacios que acerquen a la sociedad con las tradiciones, los saberes ancestrales y la ciencia. Científicos de diferentes áreas han llegado a la conclusión de que la conservación de la biodiversidad 


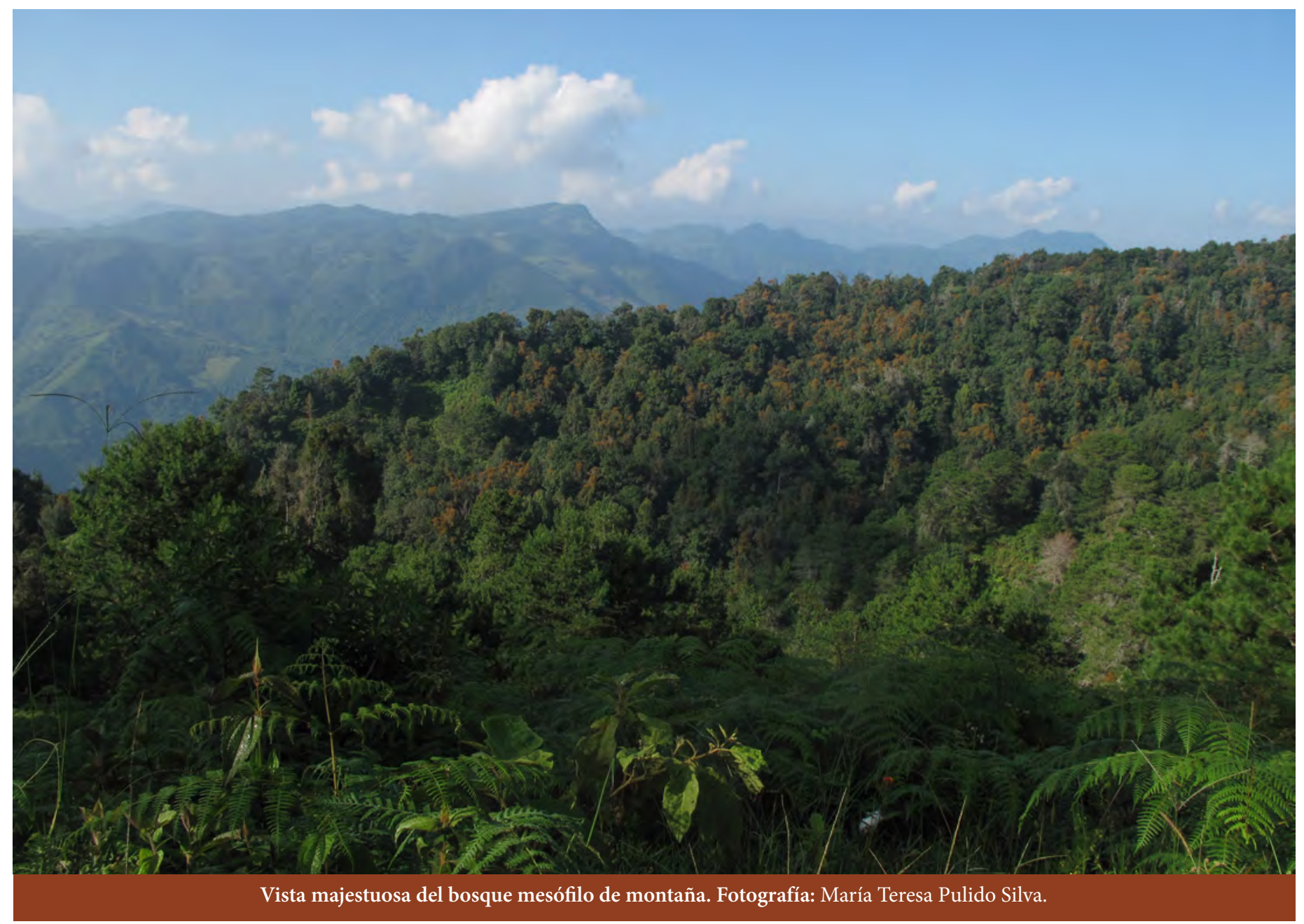

se va a lograr cuando cambiemos nuestra forma occidental de entender el mundo y aprendamos de los pueblos originarios que han sabido mantener su riqueza biológica gracias a que su cosmovisión se basa en el respeto a la flora, la fauna, el agua y el suelo. Uno de ellos, el etnoecólogo Víctor Manuel Toledo, ha comparado las zonas del planeta en las que hay una mayor diversidad cultural con las regiones que albergan los mayores números de especies y de endemismos y ambas se sobrelapan. Es decir, nueve de los países con la mayor riqueza en el número de especies están en la lista de los 25 que tienen una mayor diversidad de pueblos indígenas (Toledo, 2010). Por su parte, Boaventura de Souza Santos reconoce que la mayor diversidad biológica del planeta se encuentra en los territorios históricamente ocupados por los pueblos indígenas y campesinos, quienes intentan garantizar su conservación y la de su forma de vida y saberes tradicionales ante el embate de los intereses de los países desarrollados tecnológicamente (De Souza Santos, 2012). Biólogos de la conservación como Richard Primack, Ricardo Rozzi y Peter Feinsinger (2001) también han señalado que las culturas indígenas "ofrecen una variedad de sofisticadas prácticas ambientales, complejas regulaciones sociales y cosmogonías que involucran el respeto de todos los seres vivos", lo que puede servir de guías éticas para que la sociedad occidental aprenda a convivir con su medio de tal manera que empiece a recuperar la terrible degradación que sus prácticas han llevado al planeta.
Esta inextricable relación entre la diversidad biológica y la cultural es lo que se ha llamado el "Concepto de conservación simbiótica" (Nietschmann, 1992, citado por Toledo et al., 2019) que señala que "la diversidad biológica y la cultural son mutuamente dependientes y geográficamente coexistentes", a lo cual también se le ha denominado el axioma biocultural. Esta correlación entre ambas diversidades tiene implicaciones de fondo en la forma en que debe realizarse la conservación, pues van de la mano. Debemos repensar la conservación de la biodiversidad teniendo en cuenta el profundo significado de esto en la práctica, pues hay implicaciones no solo biológicas, sino también -y de manera esencial- sociales y debe ser comprendida a través de la mirada de diferentes culturas. Estos preceptos deberán ser mostrados en los diferentes jardines etnobiológicos que se establezcan en el país.

Un jardín etnobiológico es conceptualmente más amplio que un jardín botánico, puesto que busca no solo conservar a las plantas y organismos de otros reinos, sino promover y fomentar el conocimiento sobre el uso y mantenimiento de esa biodiversidad. El conocimiento humano puede obtenerse por distintos caminos, uno de los cuales es la ciencia, otro es el conocimiento tradicional. Este se refiere a un conjunto complejo de profundas reflexiones teórico prácticas que hace la gente de sociedades pre-industriales en muchas culturas que por siglos o en ocasiones por milenios han desarrollado 
un complejo entendimiento de la naturaleza y del universo. Este conocimiento tradicional se exhibe en varias dimensiones: los organismos (nombres comunes, sistemas de clasificación taxonómicas), las relaciones entre estos (redes tróficas) y con su ambiente (procesos geofísicos, ciclos hidrológicos), los procesos (de sucesión, de floración), entre otros. El conocimiento tradicional se caracteriza básicamente por ser colectivo, por su análisis conjunto (holístico) y por transmitirse de manera oral entre generaciones. Es por esto que actualmente el conocimiento tradicional enfrenta fuertes amenazas, pues se expone a fenómenos como la migración, la globalización y la subvaloración.

Una manera de hacer contrapeso a esta realidad es el fomento de los jardines etnobiológicos donde se disponga de espacios que permitan la expresión, el intercambio y el aprendizaje de esos conocimientos tradicionales, a la vez que permita la conservación in situ y ex situ de especies de importancia cultural. A la fecha no existe un jardín botánico en Hidalgo y es esta otra de las razones por las que el Jardín Etnobiológico Maximino Martínez será de importancia para la región.

Mientras que el estado de Hidalgo solo ocupa el $1 \%$ de la superficie del país, su diversidad biológica y cultural es muy alta, por lo que en esta primera fase se plantearon dos sedes contrastantes y complementarias:

\section{Tlanchinol}

La vegetación que predomina en este municipio es el bosque mesófilo de montaña (BMM), uno de los ecosistemas de mayor biodiversidad del mundo y el cual es catalogado como un ecosistema en peligro de extinción (Piedra, 2016); también hay bosque tropical perennifolio y bosque de coníferas en menor proporción. El BMM es muy relevante por la diversidad de especies que alberga por unidad de superficie, además de los servicios ambientales que provee incluyendo el mantenimiento de los ciclos hidrológicos (Piedra, 2016). En Tlanchinol casi el 84\% de la población se considera indígena por conservar sus tradiciones y 50\% habla el náhuatl, además del castellano (PMD, 2020).

En Tlanchinol el BMM se ha transformado debido a factores políticos, económicos y sociales. La escasez internacional de manganeso hacia 1950 llevó al aprovechamiento de minas de este mineral en Molango (contiguo a Tlanchinol) y Nonoalco. Varios de los trabajadores de estas minas se fueron a vivir a Tlanchinol, lo que causó que el pueblo creciera notablemente y con ello la demanda de recursos sobre el BMM. Por otra parte, también por una demanda internacional, el gobierno financió y promovió la realización de proyectos ganaderos entre 1960 y 1970, llegando a desmontar superficies importantes de bosque (Piedra, 2016). Recientemente los comuneros han recibido apoyos gubernamentales de pago por servicios ambientales lo que indiscutiblemente ha ayudado a su conservación. Sin embargo, uno de los grandes desafíos es lograr una gobernanza más sólida al interior de la localidad.
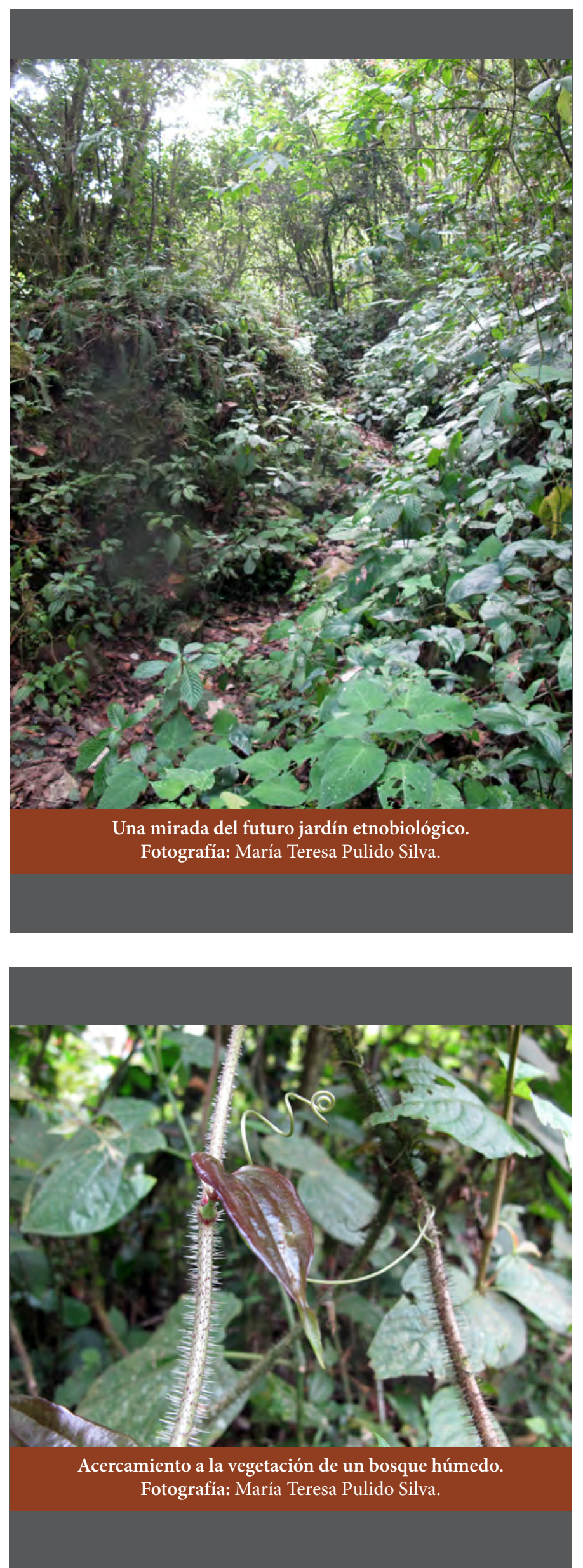


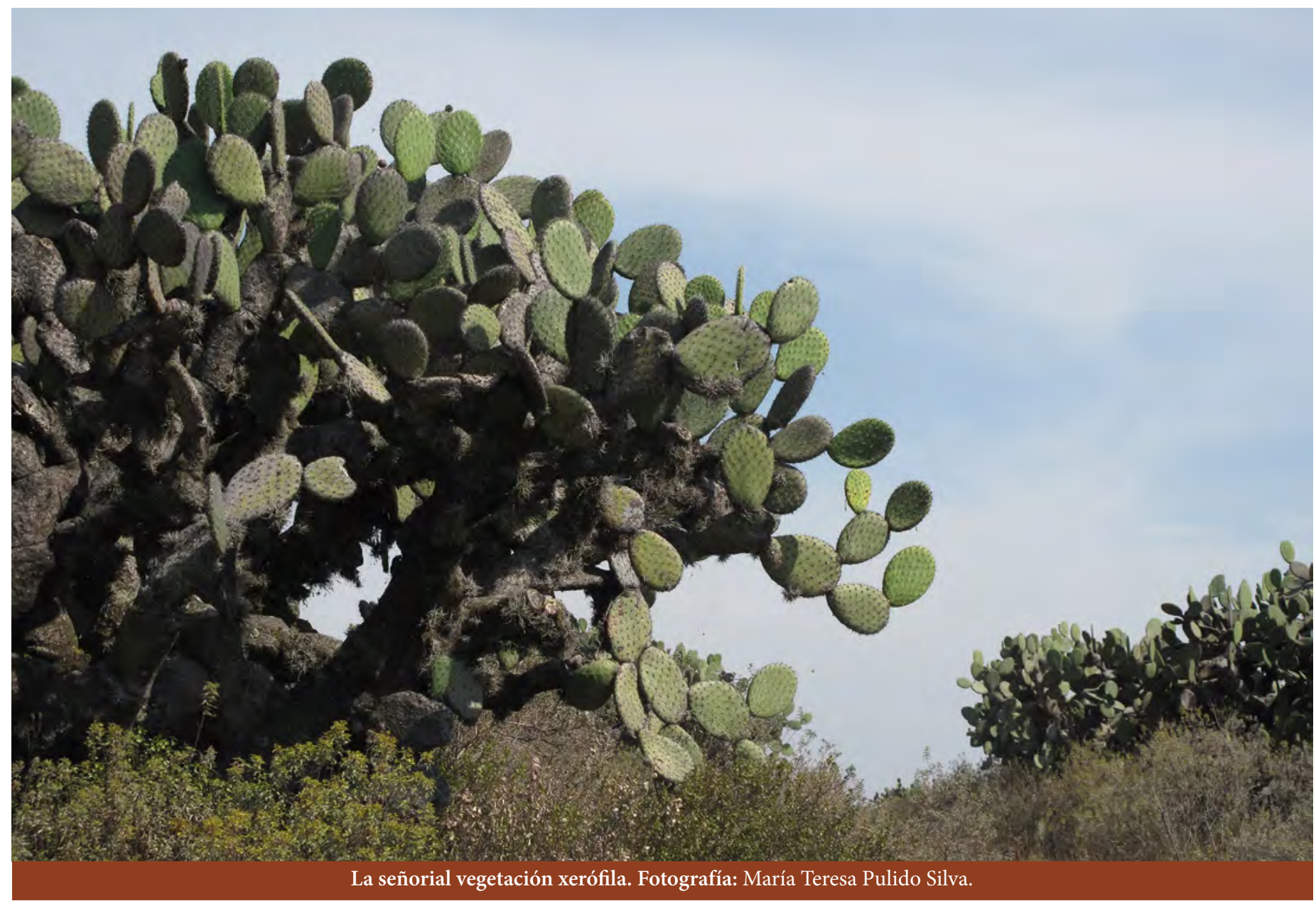

\section{Pachuca}

La otra sede del Jardín Etnobiológico será la ciudad de Pachuca, en donde se encuentran los terrenos del Parque Científico y Tecnológico de nuestra Universidad. Aunque Pachuca actualmente cuenta con una extensión urbana que cubre la mayor parte de su territorio, hasta hace muy pocos años esto no era así. Como es bien sabido, la ciudad de Pachuca se fundó debido a sus minas en el siglo XVI. Sin embargo, sus alrededores fueron ocupados por tierras de cultivo de diferentes productos, principalmente maguey, maíz, frijol, chile y calabaza. Durante siglos fue una zona de alta producción del pulque que se llevaba a las minas y también a la Ciudad de México.

En 1856 se estableció la Ley de Desamortización de Bienes y en 1875 la Ley General de Colonización, lo que llevó a que se les arrebataran a los pueblos las tierras comunales, por considerar que si no tenían dueño podían pasar a formar parte de las haciendas en las que había un propietario y en donde supuestamente serían más productivas. Así se fueron formando haciendas de grandes extensiones en Pachuca, como La Concepción, San Ignacio, San Javier, El Palmar, Coscotitlán, Pitahayas y Santa Matilde. Varias de estas pertenecían a herederos descendientes de Pedro Romero de Terreros, el famoso conde de Regla. A raíz de la Revolución mexicana y después del reparto agrario, entre 1921 y 1938, los pueblos solicitaron restitución o dotación de tierras y se formaron nueve ejidos en el municipio: Santiago Tlapacoya, Aquiles Serdán, Venta Prieta, San Antonio, Cerezo, Santa Julia,
Huixmí, Santa Matilde y San Cayetano (Cuevas-Cardona, 1993). El primer poblado en pedir restitución de tierras fue el de Santiago Tlapacoya, justo en donde se va a instalar el jardín etnobiológico. Sin embargo, todos comparten una historia común y una sabiduría ancestral, que es esencial dar a conocer en el jardín para conformar nuestra historia y recuperar esos saberes.

Ambas sedes representan dos realidades muy contrastantes: mientras que la de Pachuca está en una zona árida, enclavada en el Valle de México, la sede de Tlanchinol es húmeda y se ubica en la Sierra Madre Oriental. De este modo hay una complementariedad entre ellas.

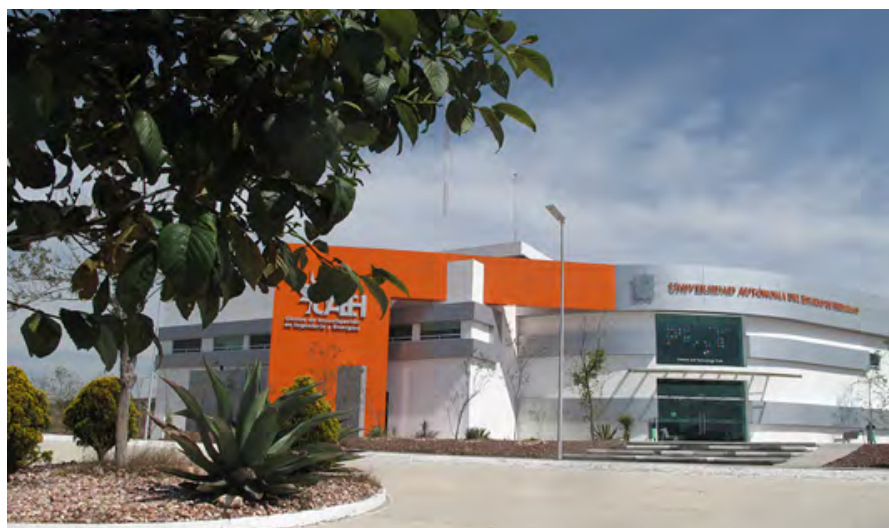

Entrada al Parque Científico y Tecnológico, sede del Jardín Etnobiológico de Pachuca. Fotografía: María Teresa Pulido Silva. 


\section{Formas de vincularse con el Jardín}

La sociedad mexicana e hidalguense, así como la comunidad estudiantil está invitada a participar de una manera activa con este jardín. Pueden comunicarse a jardinetnobiologico@gmail. com y muy pronto también podrán hacerlo mediante una página web en la que se dará a conocer el programa de actividades. Además, los alumnos se pueden vincular para desarrollar sus tesis de licenciatura, prácticas profesionales o servicio social.

\section{Referencias}

Cuevas Cardona, M. C. 1993. Historia y ecología de los ejidos de Pachuca. Gobierno del Estado de Hidalgo, Pachuca.

De Souza Santos, B. 2012. Una epistemología del Sur. CLACSO/Siglo XXI editores, México.

Nietschmann, B. Q. 1992. The interdependence of biological and cultural diversity. Occas, Papers 21. Center of World Indigenous Studies, Olympia.

Piedra, D. K. 2016. Componentes socio-ambientales de Bienes Comunales del municipio de Tlanchinol (Hidalgo), y sus implicaciones en la conservación del bosque mesófilo de montaña. Un enfoque de participación comunitaria. Tesis de Maestría en Biodiversidad y Conservación, Universidad Autónoma del Estado de Hidalgo.

PMD. 2020. Plan Municipal de Desarrollo de Tlanchinol 2016-2020. [https://www.tlanchinol.gob.mx/contenidos/tlanchinol/ docs/16_plan-municipal-tlanchinol-2016-2020_20427165139. pdf].

Primack, R., Rozzi, R., Feinsinger, P., Dirzo, R. y Massardo, F. 2001. Fundamentos de conservación biológica. Perspectivas latinoamericanas. Fondo de Cultura Económica, México.

Toledo, V. M. 2010. Ecología, espiritualidad, conocimiento. Jitanjáfora Morelia Editorial, Morelia.

Toledo, V. M., Barrera-Bassols, N. y Boegle, E. 2019. ¿Qué es la diversidad biocultural? Universidad Nacional Autónoma de México, en coedición con la Red para el Patrimonio Biocultural, Conacyt, México.

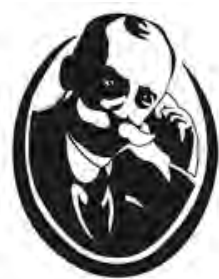

